of assessing the sensitivity and specificity of such definitions in an objective manner, and of reaching a consensus on any change in their use.

Original article Kuban D et al. (2004) Failure definitiondependent differences in outcome following radiation for localized prostate cancer: can one size fit all? Int J Radiat Oncol Biol Phys 61: 409-414

\section{Chlorambucil and lomustine (CL56) to treat androgen- independent prostate cancer}

A recent paper by Shamash and colleagues describes the treatment of absolute hormone refractory prostate cancer using a simple oral chemotherapy regimen. Despite a low PSA response rate, there was evidence of antitumor activity in approximately two-thirds of patients and, surprisingly, hormone sensitivity returned in several patients after treatment.

This phase II trial included 37 patients with advanced, end-stage prostate cancer. The men received up to six cycles of a combination of chlorambucil and lomustine (CL56), having failed androgen blockade and secondline hormonal therapy. All hormone therapy was stopped for the duration of treatment and steroids were reduced where possible.

The authors comment that the objective response rate of $10 \%$ was modest. PSA was normalized in one patient and reduced by more than $50 \%$ in two others. Unexpectedly, however, hormone sensitivity returned in 8 of 17 non-responders who went on to receive further hormone treatment, and 3 of these patients had a further PSA decrease of more than $50 \%$. The chemotherapy regimen was well tolerated; grade 3 malaise, constipation, thrombocytopenia and leukopenia were the most serious toxicities.

The authors comment that survival (a median of 23.5 months from becoming androgen independent) compared favorably with taxane/ estramustine combinations, which are less well tolerated. They stress the need for prospective studies of the observed reinduction of hormone sensitivity.

Original article Shamash J et al. (2005) Chlorambucil and lomustine (CL56) in absolute hormone refractory prostate cancer: re-induction of endocrine sensitivity an unexpected finding. Br J Cancer 92: 36-40

\section{A guide to grant writing for the clinical researcher}

Inouye and Fiellin, from Yale University School of Medicine, have recently provided an evidencebased guide to grant writing for the new clinical researcher. They state that application of the systematic approach described should help researchers to manage this important task.

Published in Annals of Internal Medicine, the guide begins by providing background information on funding sources, the types of grants available, and the $\mathrm{NIH}$ review process. Examples of timelines are presented, detailing the steps involved in the grant-writing process, from the conceptualization of the project and initiation of pilot work to submission of the final grant.

Having analyzed review sheets from $66 \mathrm{NIH}$ clinical research grant applications, Inouye and Fiellin defined the areas most often commented on by reviewers. The specific aims and hypotheses, although judged to be the most important part of the grant, are often 'poorly focused, underdeveloped, or overly ambitious'. Preliminary work is often lacking or is not well described, and applicants frequently fail to describe the significance with regard to the proposed study. The Methods section of the application receives much of the criticism from reviewers, usually because the methods are underdeveloped and fail to include sufficient details on design and setting, inclusion and exclusion criteria, availability of participants, data collection procedures, the intervention strategy itself, and data analysis. Inouye and Fiellin provide guidance on all these aspects, as well as a checklist covering each section of the grant.

Although geared towards clinical research and to $\mathrm{NIH}$ funding in particular, this paper should also be useful to those seeking funding elsewhere and to scientists in general.

Original article Inouye SK and Fiellin DA (2005) An evidence-based guide to writing grant proposals for clinical research. Ann Intern Med 142: 274-282

\section{EphA2 expression predicts outcome in patients with renal cell carcinoma}

The receptor tyrosine kinase EphA2 is overexpressed in some epithelial carcinomas, and in metastatic lesions in particular. Having studied 